\title{
Phagocytosis and intracellular killing of heterogeneous vancomycin-intermediate Staphylococcus aureus strains
}

Correspondence

László Maródi

Imarodi@dote.hu

Received 4 January 2011

Accepted 22 September 2011
Leonóra Méhes, ${ }^{1}$ Szilvia Taskó, ${ }^{1}$ Annamária Székely, ${ }^{1}$ Ákos Tóth, ${ }^{2}$ Erika Ungvári, ${ }^{3}$ Irén Erdei, ${ }^{4}$ Zsuzsanna Dombrádi, ${ }^{5}$ Judit Szabó ${ }^{5}$ and László Maródi ${ }^{1}$

${ }^{1}$ Department of Infectious and Pediatric Immunology, Medical and Health Science Center, University of Debrecen, Debrecen, Hungary

${ }^{2}$ Department of Bacteriology, National Center for Epidemiology, Budapest, Hungary

${ }^{3}$ Department of Phage and Molecular Typing, National Center for Epidemiology, Budapest, Hungary

${ }^{4}$ Department of Dermatology, Medical and Health Science Center, University of Debrecen, Debrecen, Hungary

${ }^{5}$ Department of Medical Microbiology, Medical and Health Science Center, University of Debrecen, Debrecen, Hungary

Risk factors for invasive infections by heterogeneous vancomycin-intermediate Staphylococcus aureus (hVISA) may involve resistance to opsonophagocytosis and bacterial killing. hVISA strains typically have a thickened cell wall with altered peptidoglycan cross-linking. To determine whether hVISA may be endowed with an increased resistance to phagocytosis, this study assessed the characteristics of uptake and killing by granulocytes of three hVISA strains. All isolates were analysed by multilocus sequence typing and staphylococcal chromosome cassette mec typing. One of the strains belonged to the Hungarian meticillin-resistant S. aureus (MRSA) clone ST239MRSA-III and the other two to the New York/Japan MRSA clone ST5-MRSA-II. In the presence of $10 \%$ normal serum, the extent of phagocytosis and killing by blood granulocytes was equivalent for hVISA, MRSA and meticillin-sensitive S. aureus (MSSA) strains. Using granulocytes and serum from one patient who survived hVISA infection, the rate of phagocytosis and killing was also found to be comparable to that by control cells in the presence of $10 \%$ serum. However, phagocytosis and killing of hVISA and MRSA (ATCC 25923) strains by normal granulocytes was markedly decreased in the presence of low concentrations (1 and $2.5 \%$ ) of serum from the patient who survived hVISA infection compared with that found with normal human serum. These data suggest that hVISA and MRSA isolates may be more resistant to opsonophagocytosis and bacterial killing than MSSA isolates, at least in some cases.

\section{INTRODUCTION}

Over the past decade, infections by meticillin-resistant Staphylococcus aureus (MRSA) strains have become increasingly prevalent, and vancomycin has remained the drug of choice for treatment of patients with MRSA infections (Garnier et al., 2006; Jones, 2006; Gould, 2008). However, due to selective pressure, $S$. aureus isolates with decreased susceptibility to vancomycin have emerged (Song et al.,

Abbreviations: AUC, area under the curve; CLSI, Clinical and Laboratory Standards Institute; hVISA, heterogeneous vancomycin-intermediate S. aureus; MLST, multilocus sequence typing; MRSA, meticillin-resistant S. aureus; MSSA, meticillin-sensitive $S$. aureus; PAP, population analysis profile; SCC, staphylococcal chromosomal cassette.
2004). The Clinical and Laboratory Standards Institute (CLSI) designates staphylococci requiring concentrations of vancomycin $\leqslant 2 \mathrm{mg} \mathrm{l}^{-1}$ for growth inhibition as susceptible, those requiring 4-8 $\mathrm{mg} \mathrm{l}^{-1}$ as intermediately sensitive and those requiring $\geqslant 16 \mathrm{mg} \mathrm{l}^{-1}$ as resistant (CLSI, 2009). Heterogeneous vancomycin-intermediate $S$. aureus (hVISA) strains appear to be sensitive to vancomycin with a susceptibility range of $1-2 \mathrm{mg} \mathrm{l}^{-1}$ but contain subpopulations of organisms with intermediate sensitivity to vancomycin (Garnier et al., 2006; Sakoulas et al., 2006). The first staphylococcal infection caused by hVISA was detected in Japan, which was followed by reports of a number of cases from other countries (Goldblum et al., 1978; Hiramatsu et al., 1997a, b; Garnier et al., 2006; Gould, 2008). Research 
on phagocytosis and killing by professional phagocytes may provide new insight into the pathogenicity of hVISA strains. We report here on three patients with severe, invasive hVISA infection. We propose that hVISA strains, as well as MRSA strains, may be more resistant than meticillin-sensitive $S$. aureus (MSSA) strains to opsonophagocytosis and killing by granulocytes.

\section{METHODS}

\section{Patients}

Patient 1. This 56-year-old man was diagnosed with a parapharyngeal abscess requiring incision and tracheotomy. Two days after admission, haemodialysis was started because he developed sepsis and acute renal failure and he remained dialysis dependent. Three months later, his jugular vein catheter was removed because of bacterial colonization and amikacin administration was started. Routine nasal and throat cultures and repeated blood cultures yielded MRSA that was susceptible to vancomycin; therefore, amikacin was combined with vancomycin and later with rifampicin. A chest X-ray revealed bilateral basal pneumonia and computed tomography revealed multiple pulmonary and spleen abscesses. Progression of the infection, ventricular arrhythmia and circulatory failure led to a fatal outcome. Post-mortem blood culture yielded MRSA with reduced vancomycin susceptibility.

Patient 2. This patient had Little's disease complicated by tetraplegia, somatomental retardation and epilepsy. She was admitted to hospital aged 24 years with pneumonia and acute respiratory failure after an epileptic attack. Empiric antibiotic therapy with clindamycin and ceftriaxone was started and a tracheotomy was performed. Pseudomonas aeruginosa and Candida species were detected in bronchial secretions and the antibiotic regimen was changed to ceftazidime, amikacin and fluconazole. In addition, parenteral nutrition had to be instituted. Later, nasal culture, blood culture and bronchial secretions yielded MRSA and treatment with vancomycin was started. The strain isolated from the bronchial culture showed reduced susceptibility to vancomycin and was confirmed as hVISA. She developed sepsis and bone marrow failure and died, despite 3 months of intensive treatment.

Patient 3. This 27-year-old woman was admitted to hospital for burns over $\sim 40 \%$ of the total body surface area. Repeated necrotomy and skin grafting were performed. During hospitalization, she developed pneumonia. Samples were taken for culture from the throat, nose, wounds and genitalia. hVISA was cultured from skin lesions and she was treated with tigecycline. Two months after admission, she was discharged from hospital with healed skin lesions.

Antibiotic susceptibility testing. Susceptibility to different antibiotics was tested by the disc diffusion method with Oxoid discs according to CLSI guidelines (CLSI, 2009; Szabó et al., 2009). Meticillin resistance was determined by oxacillin Etest (AB Biodisk). MICs of vancomycin, teicoplanin, erythromycin, clindamycin, ciprofloxacin, gentamicin, amikacin, rifampicin, tigecycline, linezolid, daptomycin and quinupristin/dalfopristin were defined by Etest according to CLSI guidelines (Brown et al., 2005; Tóth et al., 2008; CLSI, 2009; Szabó et al., 2009). The suspected hVISA strains were examined using the macro Etest method (Tóth et al., 2008).

Population analysis profile (PAP). Fifty microlitres of the starting bacterial cell suspension (prepared by diluting a culture grown overnight to an $\mathrm{OD}_{578}$ of 0.3 ) was diluted to $1: 10$ and $1: 10^{4}$ and spread on brain-heart infusion agar plates (Oxoid) containing $0,0.5$,
$1.0,2.0,2.5,4$ and $8 \mathrm{mg}$ vancomycin $1^{-1}$. Colonies were counted after $48 \mathrm{~h}$ of incubation at $37^{\circ} \mathrm{C}$ using a semi-logarithmic graph of counted c.f.u. versus vancomycin concentration. The area under the curve (PAP-AUC) was calculated for each strain (Walsh et al., 2001). To distinguish VISA, hVISA and vancomycin-susceptible MRSA (ATCC 29213), a ratio of the AUC of the test MRSA divided by the corresponding AUC for $S$. aureus strain Mu3 used as a positive control was calculated. The criteria used for detection of hVISA and VISA strains were AUC ratios of $\geqslant 0.9$ and $\geqslant 1.3$, respectively. The ratio of test MRSA AUC divided by the corresponding Mu3 AUC was calculated as described previously (Walsh et al., 2001; Tóth et al., 2008).

Molecular typing methods. The hVISA/VISA strains were typed by multilocus sequence typing (MLST) and staphylococcal chromosome cassette (SCC) mec typing (Enright et al., 2000; Oliveira \& de Lencastre, 2002). Allele sequences and sequence types were verified at the http://www.mlst.net website.

Granulocytes. Granulocytes were separated from heparinized (10 U $\mathrm{ml}^{-1}$ ) venous blood as described previously (Maródi et al., 1984, 2001). Cells were washed and resuspended to a concentration of $10^{7}$ $\mathrm{ml}^{-1}$ in Krebs-Ringer phosphate buffer with $5 \%$ glucose (KRPG). The granulocyte suspension contained $>98 \%$ neutrophils (band form and segmented) as demonstrated by May-Grünwald-Giemsa staining in cytocentrifuge preparations. Cells were finally resuspended in KRPG to a concentration of $10^{7}$ cells $\mathrm{ml}^{-1}$.

Preparation of sera. Whole blood was obtained from eight healthy adults. Blood was allowed to clot at room temperature for $1 \mathrm{~h}$, followed by centrifugation at $4{ }^{\circ} \mathrm{C}$ (Maródi et al., 1993). Serum was removed and stored in aliquots at $-20{ }^{\circ} \mathrm{C}$ until use (Maródi et al., 1998).

Bacteria. MSSA (ATCC 43300), MRSA (ATCC 25923) and three hVISA isolates from patients 1,2 and 3 were cultured overnight at $37{ }^{\circ} \mathrm{C}$ in $100 \mathrm{ml}$ nutrient broth (Oxoid). Bacteria were harvested by centrifugation at $1500 \mathrm{~g}$ for $10 \mathrm{~min}$, washed twice with KRPG and resuspended in KRPG to a concentration of $10^{7}$ bacteria $\mathrm{ml}^{-1}$ (Maródi et al., 1995).

Phagocytosis. Phagocytosis of bacteria by granulocytes was measured as the percentage decrease in the number of viable extracellular bacteria in the phagocytic cell suspension (Maródi et al., 1983, 2000). Briefly, $200 \mu \mathrm{l}$ granulocyte suspension $\left(10^{7}\right.$ cells $\left.\mathrm{ml}^{-1}\right)$ was incubated with $200 \mu \mathrm{l}$ bacterial suspension $\left(10^{7}\right.$ bacteria $\left.\mathrm{ml}^{-1}\right)$ in the presence of various concentrations of serum at $37{ }^{\circ} \mathrm{C}$ under slow rotation (4 r.p.m.). After 60 or $120 \mathrm{~min}$ incubation, $50 \mu \mathrm{l}$ aliquots were removed and added to $450 \mu \mathrm{l}$ cold KRPG and the cells were centrifuged for $6 \mathrm{~min}$ at 600 r.p.m. in a bench microfuge. The number of viable extracellular bacteria was determined by serial tenfold dilutions in KRPG and plating on nutrient agar plates. Colonies were counted after incubation of the plates at $37{ }^{\circ} \mathrm{C}$ for $18 \mathrm{~h}$.

Bacterial killing. Bacterial killing was measured as the percentage decrease in the number of viable extracellular and intracellular bacteria in the phagocytic cell suspension (Maródi et al., 1984, 1995). Briefly, $2 \times 10^{6}$ granulocytes (concentration $5 \times 10^{6} \mathrm{ml}^{-1}$ ) and bacteria (concentration $5 \times 10^{6} \mathrm{ml}^{-1}$ ) were incubated in the presence of various concentrations of serum at $37^{\circ} \mathrm{C}$ under slow rotation (4 r.p.m.). After 60 or $120 \mathrm{~min}$ of incubation, $50 \mu \mathrm{l}$ aliquots were removed and added to $450 \mu \mathrm{l}$ cold distilled water to disrupt the granulocytes. The total number of viable bacteria was determined by serial dilutions in KRPG and plating on nutrient agar plates. A control experiment without serum showed a 24 and $52 \%$ increase in the 


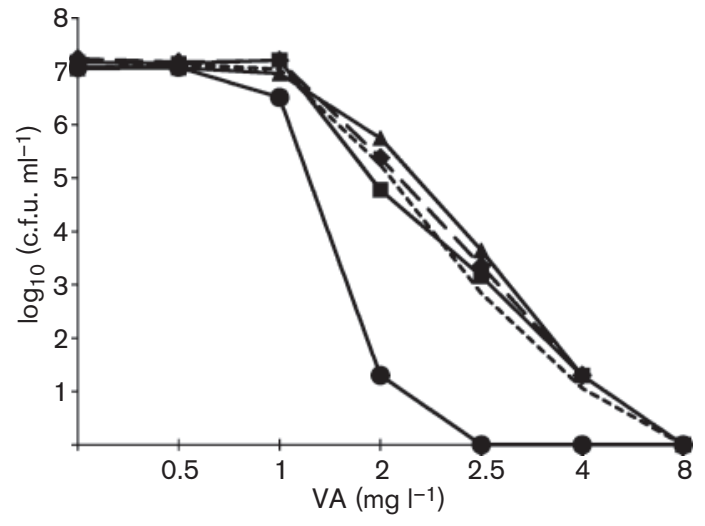

Fig. 1. Modified PAPs for the hVISA strains hVISA1, hVISA2 and hVISA3 from patients $1(\boldsymbol{\square}), 2(\mathbf{A})$ and $3(---)$, respectively. Strain Mu3 ( $)$ was used as a positive control, and MRSA ATCC 29213 (๑) was used as a negative control.

number of $S$. aureus in the presence of granulocytes over 60 and 120 min incubation, respectively.

\section{RESULTS}

\section{Antibiotic sensitivity profile of the hVISA strains}

We identified three new hVISA isolates from three different patients, which increases the number of $S$. aureus isolates with the same vancomycin resistance spectrum in Hungary to five (Tóth et al., 2008, this report, and one unpublished case). In patient 1 , the MRSA strain was isolated from blood and was confirmed by a macro Etest and population analysis as hVISA. On the basis of the macro Etest, the inhibiting concentrations of vancomycin and teicoplanin were 8 and $12 \mathrm{mg} \mathrm{l}^{-1}$, respectively. The PAP-AUC ratio was 1.01 as shown in Fig. 1. The MIC values for other antibiotics are shown in Table 1. According to the MLST and SCCmec typing results, this strain belonged to the Hungarian clone (ST239-MRSA-III).

The MRSA strain isolated from patient 2 was shown to be hVISA by macro Etest and population analysis. Inhibiting concentrations of vancomycin and teicoplanin were both
$8 \mathrm{mg}^{-1}$. The PAP-AUC ratio was 0.97 (Fig. 1). The MIC values for other antibiotics are listed in Table 1. The isolate was also tested by MLST and SCCmec typing, and was found to belong to the New York/Japan MRSA clone (ST5MRSA-II).

The MRSA strain isolated from patient 3 also had intermediate vancomycin sensitivity according to the macro Etest. By macro Etest, the inhibiting concentrations of vancomycin and teicoplanin were both $8 \mathrm{mg} \mathrm{l}^{-1}$. The PAPAUC ratio was 0.93 (Fig. 1), confirming that it was a hVISA strain. Testing of the isolate by MLST and SCCmec typing confirmed that it also belonged to the New York/Japan MRSA clone (ST5-MRSA-II).

\section{Phagocytosis and intracellular killing of hVISA, MRSA and MSSA strains by granulocytes}

We studied the phagocytosis of opsonized hVISA, MRSA and MSSA strains by freshly isolated peripheral blood granulocytes from healthy donors. In the presence of $10 \%$ normal human serum as a source of opsonins, granulocytes displayed a comparable degree of ingestion of these strains after $60 \mathrm{~min}$ incubation (Table 2). Over a period of $120 \mathrm{~min},>90 \%$ of bacteria were ingested, independent of their antibiotic sensitivity.

We also compared the killing of hVISA, MRSA and MSSA strains by blood granulocytes in the presence of $10 \%$ serum over a period of $120 \mathrm{~min}$. Similar to the uptake of staphylococcal strains with different susceptibility for antibiotics, killing of these bacteria by normal granulocytes was also comparable at both 60 and $120 \mathrm{~min}$ time points (Table 2). The opsonic activity of serum from patient 3 , who survived the hVISA infection, was tested on two occasions over a 2-month period. The ingestion and killing by normal granulocytes of hVISA at 60 and $120 \mathrm{~min}$ in the presence of $10 \%$ normal human serum or $10 \%$ serum from patient 3 was comparable (Fig. 2). Phagocytosis and killing of hVISA by the patient's granulocytes in the presence of $10 \%$ normal serum was also comparable to that mediated by the patient's serum of normal granulocytes.

Next, we compared phagocytosis and killing of hVISA, MRSA and MSSA by normal granulocytes in the presence

Table 1. MICs for the hVISA strains isolated from the patients in this study

Oxa, Oxacillin; Ery, erythromycin; Cli, clindamycin; Cip, ciprofloxacin; Gen, gentamicin; Ami, amikacin; Rif, rifampicin; Tig, tigecycline; Dap, daptomycin; Qui/Dal, quinupristin/dalfopristin; Lin, linezolid.

\begin{tabular}{|c|c|c|c|c|c|c|c|c|c|c|c|}
\hline \multirow[t]{2}{*}{ hVISA } & \multicolumn{11}{|c|}{$\operatorname{MIC}\left(\mathrm{mg} \mathrm{l}^{-1}\right)$} \\
\hline & Oxa & Ery & Cli & Cip & Gen & Ami & Rif & Tig & Dap & Qui/Dal & Lin \\
\hline hVISA1 & $>256$ & $>256$ & $>256$ & $>32$ & 24 & 16 & 0.006 & 0.50 & 1 & 1 & 1.5 \\
\hline hVISA2 & $>256$ & $>256$ & $>256$ & $>32$ & 64 & 64 & $>32$ & 0.50 & 1 & 1 & 1 \\
\hline hVISA3 & $>256$ & $>256$ & $>256$ & $>32$ & 32 & 16 & 0.006 & 0.25 & 1 & 2 & 0.75 \\
\hline
\end{tabular}


Table 2. Comparison of phagocytosis and killing of $S$. aureus strains with varying antibiotic sensitivities by peripheral blood granulocytes

Phagocytosis and bacterial killing of MSSA (ATCC 43300), MRSA (ATCC 25923) and hVISA strains by peripheral blood polymorphonuclear neutrophil granulocytes from healthy donors. Phagocytic cells were incubated for 120 min with bacteria in the presence of $10 \%$ normal human serum. Data represent means \pm SEM of at least three experiments.

\begin{tabular}{|lccccc|}
\hline S. aureus strain & \multicolumn{2}{c}{ Phagocytosis $\mathbf{( \% )}$} & & \multicolumn{2}{c|}{ Staphylococcal killing (\%) } \\
\cline { 2 - 3 } \cline { 5 - 5 } & $\mathbf{6 0} \mathbf{~ m i n}$ & $\mathbf{1 2 0} \mathbf{~ m i n}$ & & $\mathbf{6 0 ~} \mathbf{~ m i n}$ & $\mathbf{1 2 0} \mathbf{~ m i n}$ \\
\hline ATCC 43300 & $92 \pm 3$ & $97 \pm 2$ & & $82 \pm 2$ & $85 \pm 4$ \\
ATCC 25923 & $85 \pm 4$ & $95 \pm 3$ & & $76 \pm 5$ & $87 \pm 6$ \\
hVISA1 & $82 \pm 3$ & $93 \pm 3$ & & $71 \pm 6$ & $80 \pm 7$ \\
hVISA2 & $80 \pm 5$ & $94 \pm 4$ & & $79 \pm 3$ & $83 \pm 5$ \\
hVISA3 & $81 \pm 6$ & $92 \pm 4$ & & $77 \pm 6$ & $86 \pm 4$ \\
\hline
\end{tabular}

of low concentrations of serum ( 1 and $2.5 \%$ ) as a source of opsonins from patient 3 and healthy individuals, respectively (Fig. 3). We found that the extent of both ingestion and killing of hVISA and MRSA was markedly decreased compared with that found with MSSA (Fig. 3). These data indicated that the capacity of patient 3's serum to promote killing of hVISA and MRSA by blood granulocytes was impaired.

\section{DISCUSSION}

We reported recently that the prevalence of MRSA in patients with invasive staphylococcal infections was as high as $25 \%$ in Hungary, and hVISA strains have emerged that may have been the result of the extensive use of glycopeptides, especially vancomycin, for the treatment of patients with severe MRSA infections (Tóth et al., 2008; Szabó, 2009). hVISA infections are usually associated with poor disease outcome with a mortality rate of $\sim 75 \%$ (Szabó, 2009). Risk factors for the emergence of hVISA/ VISA strains may include haemodialysis, burn wounds and prolonged use of indwelling foreign devices (Goldblum et al., 1978; Boelaert, 1994; Szabó, 2009). In the patients described in this report, chronic haemodialysis, prolonged use of indwelling catheters and severe burns, respectively, may have contributed to the occurrence of invasive hVISA infection. In each case, MRSA nose colonization might have also contributed to the emergence of hVISA.

hVISA strains have been reported from a number of countries, and most of the isolates belonged to the New York/Japan MRSA clone (CDC, 1997; Hiramatsu et al., 1997a, b; Ploy et al., 1998). To date, five cases of hVISA infection have been detected in Hungary. Two of the hVISA strains described in this report belonged to the New York/Japan MRSA clone ST5-MRSA-II and one to the Hungarian clone ST239-MRSA-III. We believe that if the vancomycin $\mathrm{MIC}$ is $>1 \mathrm{mg} \mathrm{l}^{-1}$, alternative therapies should be considered to avoid the possibility of treatment failure and the selection of hVISA/VISA strains.

Staphylococci display cell-bound protein A, which may interfere with opsonophagocytosis by binding the Fc part of IgG and inhibiting uptake by phagocytic cells through their Fc receptors. It has been described previously that several hVISA strains were phagocytosed by polymorphonuclear neutrophil leukocytes similarly to MRSA and

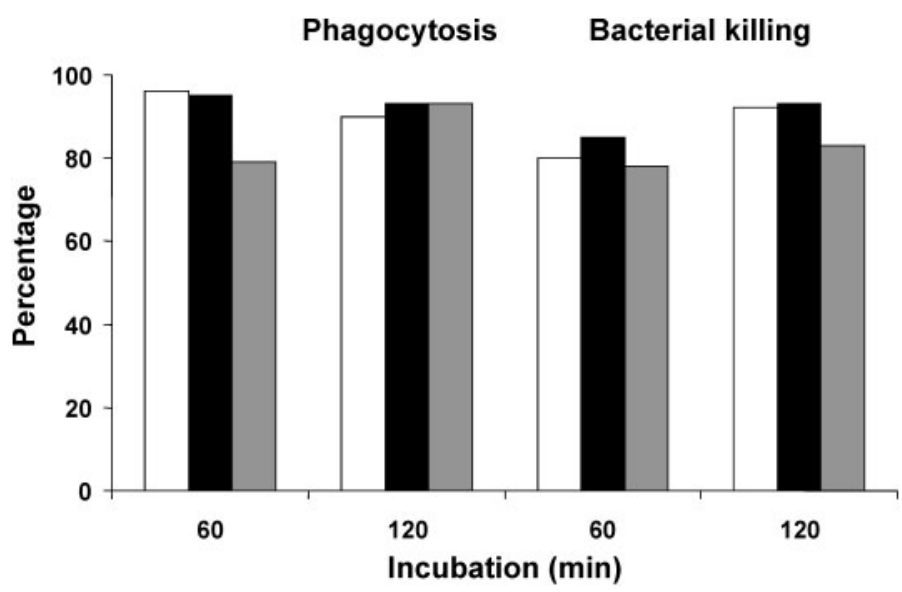

Fig. 2. Comparison of phagocytosis and killing by normal peripheral blood granulocytes of the opsonized hVISA strain isolated from patient 3. Phagocytosis (left) and bacterial killing (right) were studied by incubation of bacteria $\left(10^{7}\right.$ $\left.\mathrm{ml}^{-1}\right)$ with normal granulocytes $\left(10^{7} \mathrm{ml}^{-1}\right)$ and $10 \%$ serum from patient 3 (open columns), normal granulocytes and normal serum (filled columns) or granulocytes from the patient and normal human serum (shaded columns) at 60 and 120 min of incubation under slow rotation (4 r.p.m.). The data are representative of two separate experiments (SEM $<6 \%$ for each). 

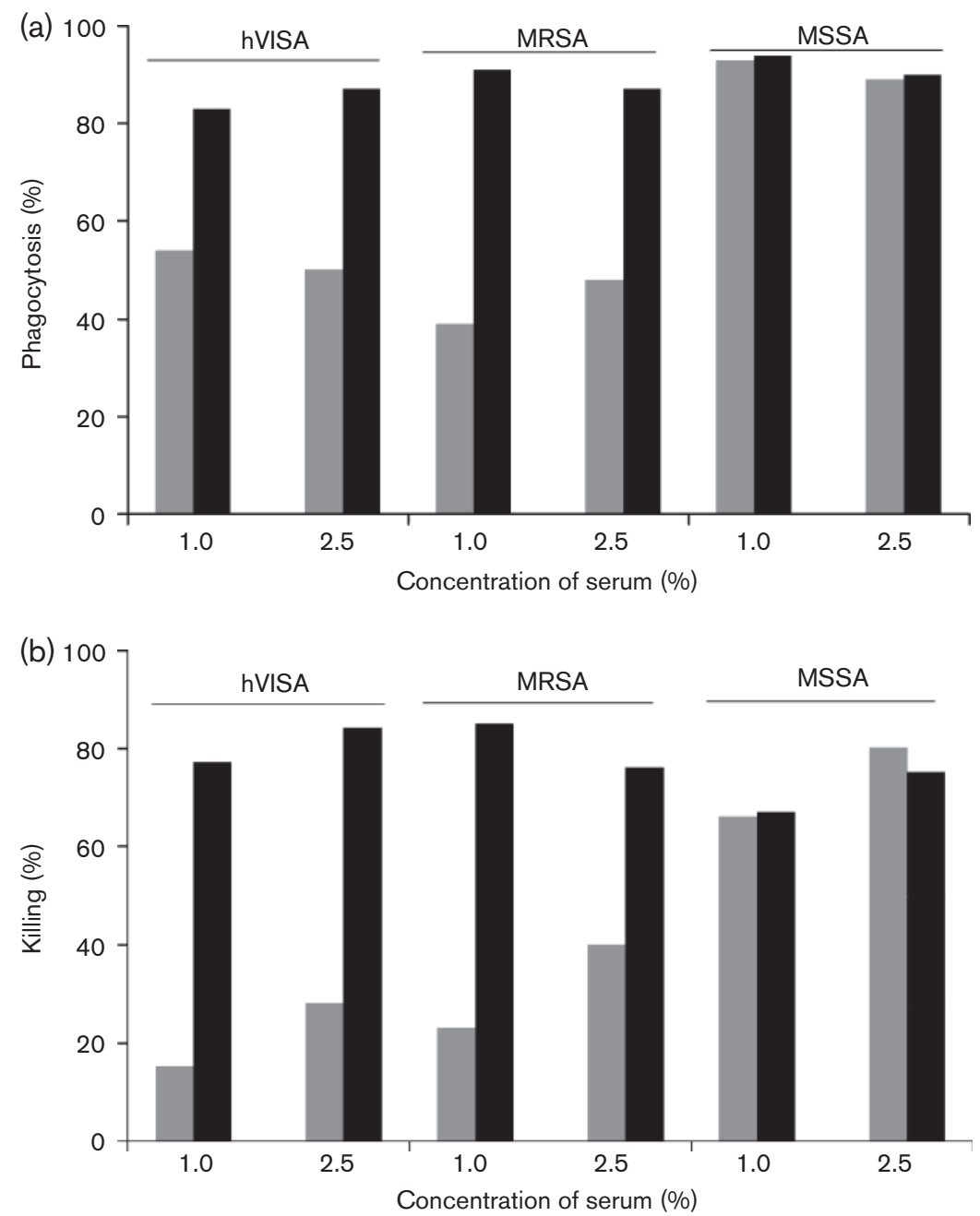

Fig. 3. Phagocytosis (a) and killing (b) of the hVISA strain isolated from patient 3 , MRSA (ATCC 25923) and MSSA (ATCC 43300) in the presence of 1 and $2.5 \%$ serum. At both concentrations, the patient's serum (shaded columns) promoted markedly decreased ingestion and killing of hVISA and MRSA compared with that found with MSSA, in contrast to the results using normal serum from a healthy individual (filled columns). The data represent means of at least three experiments performed in duplicate (SEM $<11 \%$ for each).

MSSA strains (Gemmell, 2004). Our findings with ATCC strains of MRSA and MSSA and the hVISA strains isolated from our patients further confirm that glycopeptide resistance and cell-wall thickening do not result in altered opsonophagocytosis and killing in the presence of a high concentration $(10 \%)$ of serum. However, by using more sensitive serum concentrations ( 1 and $2.5 \%$ ), we found markedly decreased phagocytic uptake and bacterial killing of hVISA and MRSA in the presence of serum from one patient with invasive hVISA infection compared with that with normal serum. The impaired capacity of the patient's serum to promote opsonophagocytosis of hVISA and MRSA strains may have contributed to the severity of the disease cause by hVISA in this case.

To our knowledge, this is the first report on intracellular killing of $S$. aureus with various antibiotic resistance profiles. In particular, we investigated the hypothesis that both hVISA and MRSA strains may be more resistant than MSSA strains to phagocytic killing by neutrophil granulocytes. This hypothesis was based on the observation that hVISA strains have a thickened cell wall with altered peptidoglycan cross-linking and a higher resistance to killing by professional phagocytes. The data presented in this report suggest that hVISA as well as MRSA strains are more resistant than MSSA strains to killing by granulocytes in the presence of low concentrations of serum. Based on these data, we propose that the structural changes in the hVISA and MRSA cell wall, which may be related to resistance to antibiotics, may also endow these staphylococci with an increased resistance to opsonophagocytosis and killing by professional phagocytes.

\section{ACKNOWLEDGEMENTS}

We thank Drs M. Zeher, K. Veres, I. Kárpáti and J. Mátyus for providing us with clinical data from their patients and P. Orosi for helpful discussion. This work was supported by TÁMOP 4.2.1./B-09/ 1/KONV-2010-0007 and TÁMOP 4.2.2-08/1-2008-0015 grants implemented through the New Hungary Development Plan and cofinanced by the European Social Fund.

\section{REFERENCES}

Boelaert, J. R. (1994). Staphylococcus aureus infection in haemodialysis patients. Mupirocin as a topical strategy against nasal carriage: a review. J Chemother 6 (Suppl. 2), 19-24. 
Brown, D. F. J., Edwards, D. I., Hawkey, P. M., Morrison, D., Ridgway, G. L., Towner, K. J. \& Wren, M. W. on behalf of the Joint Working Party of the British Society for Antimicrobial Chemotherapy, Hospital Infection Society and Infection Control Nurses Association (2005). Guidelines for the laboratory diagnosis and susceptibility testing of methicillin-resistant Staphylococcus aureus (MRSA). J Antimicrob Chemother 56, 1000-1018.

CDC (1997). Staphylococcus aureus with reduced susceptibility to vancomycin - United States, 1997. MMWR Morb Mortal Wkly Rep 46, $765-766$.

CLSI (2009). Performance Standards for Antimicrobial Susceptibility Testing, 19th Informational Supplement. M100-S19. Wayne, PA: Clinical and Laboratory Standards Institute.

Enright, M. C., Day, N. P., Davies, C. E., Peacock, S. J. \& Spratt, B. G. (2000). Multilocus sequence typing for characterization of methicillin-resistant and methicillin-susceptible clones of Staphylococcus aureus. J Clin Microbiol 38, 1008-1015.

Garnier, F., Chainier, D., Walsh, T., Karlsson, A., Bolmström, A., Grelaud, C., Mounier, M., Denis, F. \& Ploy, M. C. (2006). A 1 year surveillance study of glycopeptide-intermediate Staphylococcus aureus strains in a French hospital. J Antimicrob Chemother 57, 146-149.

Gemmell, C. G. (2004). Glycopeptide resistance in Staphylococcus aureus: is it a real threat? J Infect Chemother 10, 69-75.

Goldblum, S. E., Reed, W. P., Ulrich, J. A. \& Goldman, R. S. (1978). Staphylococcal carriage and infections in hemodialysis patients. Dial Transplant 7, 1140-1163.

Gould, I. M. (2008). Clinical relevance of increasing glycopeptide MICs against Staphylococcus aureus. Int J Antimicrob Agents 31 (Suppl. 2), 1-9.

Hiramatsu, K., Aritaka, N., Hanaki, H., Kawasaki, S., Hosoda, Y., Hori, S., Fukuchi, Y. \& Kobayashi, I. (1997a). Dissemination in Japanese hospitals of strains of Staphylococcus aureus heterogeneously resistant to vancomycin. Lancet 350, 1670-1673.

Hiramatsu, K., Hanaki, H., Ino, T., Yabuta, K., Oguri, T. \& Tenover, F. C. (1997b). Methicillin-resistant Staphylococcus aureus clinical strain with reduced vancomycin susceptibility. $J$ Antimicrob Chemother 40, 135-136.

Jones, R. N. (2006). Microbiological features of vancomycin in the 21st century: minimum inhibitory concentration creep, bactericidal/ static activity, and applied breakpoints to predict clinical outcomes or detect resistant strains. Clin Infect Dis 42 (Suppl. 1), S13-S24.

Maródi, L., Leijh, P. C. J. \& Van Furth, R. (1983). A micromethod for the separate evaluation of phagocytosis and intracellular killing of Staphylococcus aureus by human monocytes and granulocytes. J Immunol Methods 57, 353-361.

Maródi, L., Leijh, P. C. \& van Furth, R. (1984). Characteristics and functional capacities of human cord blood granulocytes and monocytes. Pediatr Res 18, 1127-1131.

Maródi, L., Schreiber, S., Anderson, D. C., MacDermott, R. P., Korchak, H. M. \& Johnston, R. B., Jr (1993). Enhancement of macrophage candidacidal activity by interferon-gamma. Increased phagocytosis, killing, and calcium signal mediated by a decreased number of mannose receptors. J Clin Invest 91, 2596-2601.

Maródi, L., Káposzta, R., Tóth, J. \& László, A. (1995). Impaired microbicidal capacity of mononuclear phagocytes from patients with type I Gaucher disease: partial correction by enzyme replacement therapy. Blood 86, 4645-4649.

Maródi, L., Tournay, C., Káposzta, R., Johnston, R. B., Jr \& Moguilevsky, N. (1998). Augmentation of human macrophage candidacidal capacity by recombinant human myeloperoxidase and granulocyte-macrophage colony-stimulating factor. Infect Immun 66, 2750-2754.

Maródi, L., Káposzta, R. \& Nemes, E. (2000). Survival of group B streptococcus type III in mononuclear phagocytes: differential regulation of bacterial killing in cord macrophages by human recombinant gamma interferon and granulocyte-macrophage colonystimulating factor. Infect Immun 68, 2167-2170.

Maródi, L., Goda, K., Palicz, A. \& Szabó, G. (2001). Cytokine receptor signalling in neonatal macrophages: defective STAT-1 phosphorylation in response to stimulation with IFN- $\gamma$. Clin Exp Immunol 126, $456-460$.

Oliveira, D. C. \& de Lencastre, H. (2002). Multiplex PCR strategy for rapid identification of structural types and variants of the mec element in methicillin-resistant Staphylococcus aureus. Antimicrob Agents Chemother 46, 2155-2161.

Ploy, M. C., Grélaud, C., Martin, C. L., de Lumley, L. \& Denis, F. (1998). First clinical isolate of vancomycin-intermediate Staphylococcus aureus in a French hospital. Lancet 351, 1212.

Sakoulas, G., Moellering, R. C., Jr \& Eliopoulos, G. M. (2006). Adaptation of methicillin-resistant Staphylococcus aureus in the face of vancomycin therapy. Clin Infect Dis 42 (Suppl. 1), S40-S50.

Song, J.-H., Hiramatsu, K., Suh, J. Y., Ko, K. S., Ito, T., Kapi, M., Kiem, S., Kim, Y.-S., Oh, W. S. \& other authors (2004). Emergence in Asian countries of Staphylococcus aureus with reduced susceptibility to vancomycin. Antimicrob Agents Chemother 48, 4926-4928.

Szabó, J. (2009). hVISA/VISA: diagnostic and therapeutic problems. Expert Rev Anti Infect Ther 7, 1-3.

Szabó, J., Dombrádi, Z., Dobay, O., Orosi, P., Kónya, J., Nagy, K. \& Rozgonyi, F. (2009). Phenotypic and genetic characterisation of methicillin-resistant Staphylococcus aureus strains isolated from the university hospitals of Debrecen. Eur J Clin Microbiol Infect Dis 28, 129-136.

Tóth, Á., Kispál, G., Ungvári, E., Violka, M., Szeberin, Z., Pászti, J., Molnár, K., Gacs, M. \& Füzi, M. (2008). First report of heterogeneously vancomycin-intermediate Staphylococcus aureus (hVISA) causing fatal infection in Hungary. J Chemother 20, 655-656.

Walsh, T. R., Bolmström, A., Qwärnström, A., Ho, P., Wootton, M., Howe, R. A., MacGowan, A. P. \& Diekema, D. (2001). Evaluation of current methods for detection of staphylococci with reduced susceptibility to glycopeptides. J Clin Microbiol 39, 2439-2444. 